Elsevier required licence: (c) <2019>. This manuscript version is made available under the CC-BY-NC-ND 4.0 license http://creativecommons.org/licenses/by-nc-nd/4.0/ The definitive publisher version is available online at https://doi.org/10.1016/j.memsci.2019.117245 


\title{
On line monitoring local fouling behavior of membrane filtration process by in situ hydrodynamic and electrical measurements
}

\author{
Hui Jia ${ }^{a, b, * *}$, Fangfang Feng ${ }^{a, b}$, Jie Wang ${ }^{a, b, * * *}$, Huu-Hao Ngo ${ }^{c, *}$, Wenshan Guo ${ }^{c}$, \\ Hongwei Zhang, \\ ${ }^{a}$ State Key Laboratory of Membrane Filtration and Processes, Tianjin Polytechnic University, Tianjin, 300387, China \\ ${ }^{\mathrm{b}}$ School of Environmental Science and Engineering, Tianjin Polytechnic University, Tianjin, 300387, China \\ ${ }^{\mathrm{c}}$ Centre for Technology in Water and Wastewater, School of Civil and Environmental Engineering, University of Technology Sydney, Sydney, NSW, 2007, Australia \\ * Corresponding author. \\ ** Corresponding author. State Key Laboratory of Membrane Filtration and Processes, Tianjin Polytechnic University, Tianjin, 300387, China. \\ ${ }^{* * *}$ Corresponding authors. State Key Laboratory of Membrane Filtration and Processes, Tianjin Polytechnic University, Tianjin, 300387, China. \\ E-mail addresses: wangjiemailbox@163.com (J. Wang), ngohuuhao121@gmail.com (H.-H. Ngo).
}

Keywords:

Hollow fiber membrane; Electrical impedance; Zeta potential; Membrane fouling; Non-uniform fouling

\section{A b s tract}

The hollow fiber ultrafiltration (UF) membrane has been widely applied in the water treatment industry, however, the membrane fouling is the core reason and limiting factor in terms of its industrial application. In the constant flux process, hollow fiber membranes (HFM) non-uniform fouling varies along the axis direction, which is the basic mechanism of HFM fouling. In this paper, the local membrane fouling behaviors and verities are investigated using electrical impedance (EI) and zeta potential (ZP) to capture the feedback signals of membrane fouling behaviors. The results are then, integrated with Hermia's model and an equivalent circuit model. As the fitting results show, both the EI and ZP can be employed as indicators of different membrane fouling states. This work defines the different stages of membrane fouling depending on the alternating relationship between EI and ZP in the membrane filtration process. Furthermore, the behavior of cake layer compaction is defined from the perspective of the membrane fouling mechanism. Therefore, this study provides an effective means for accurate identification of membrane fouling behavior. In addition, the EI and ZP exhibit great potential to identify the fouling distributions and proceedings in HFM fouling. Doing so successfully confirms that the characteristics of non-uniform fouling of HFM are reflected in the spatiotemporal difference of the fouling process.

\section{Introduction}

Due to current water shortages and problems concerning contamination, the UF process applied to the drinking water industry has undergone accelerated development in recent decades [1,2]. However, membrane fouling is still considered a crucial challenge due to the resulting severe flux decline and hence process efficiency during the pressure-driven filtration mode [3,4]. Although various operational conditions and non-uniform distribution of transmembrane pressure (TMP) could lead to membrane fouling, the most important factors are flux and filtration resistance along the axis direction of the fiber owing to the lumen-side pressure drop $[5,6]$.

Local flux, resistance and TMP along these membrane fibers are found to be of non-uniform distribution in the filtration process. The degree of non-uniform distribution constantly changes with proceeding filtration, which is affected by the structure of the hollow fiber membranes and operational conditions. In addition, the need for more energy consumption by aeration and back-washing to control membrane fouling is due to the fact that this non-uniform filtration mode accelerates the accumulation of foulant near the outlets of hollow fibers [7]. Various models have been applied to describe the local flux decrease in local flux behaviors so as to deeply understand of the fouling behaviors of hollow fiber membranes. Most of these methods are based on the Hagen-Poiseuille equations that investigate the effects of hollow fiber characteristics on local flux distributions $[7,8]$. Researchers have established a number of membrane fouling theoretical models to analyze the flux decreasing behaviors on account of membrane fouling.

Cake layer formation, intermediate blocking, standard blocking and complete blocking are the four most common membrane fouling mechanisms in the literature [9-12]. However, there are some deviations between the assumed conditions of these mathematical models and the actual experimental process. It is important to note that there is no direct evidences proving the validity of these mathematical models. Hence, local membrane fouling of HFM has been experimentally 
investigated by some researchers. Yoon et al. established an experimental method to determine the distribution of pressure drop across the membrane, which indicated that the local flux at the end is not the largest because the cake layer at this point is compressed due to the larger TMP [13]. A new experimental method was developed to study the membrane fouling distribution of hollow fiber membrane in a horizontal dead-end filtration mode. Yoon et al. indicated there was a good relationship between local membrane fouling and local TMP, specifically that the larger TMP was, where the more serious membrane fouling occurred [14]. Nonetheless, this method is cumbersome and seriously compromise the integrity of the membrane structure. For this reason it is impossible to deeply analyze the local membrane fouling behavior and the distribution of local membrane fouling from a microscopic perspective. It is required to develop an in situ and non-destructive technique so as to sensitively monitor the fouling behaviors during the membrane filtration process. Of importance is the need to fully study the correlation between local membrane fouling behaviors and non-uniform distributions, the aims being to: firstly, confirm the results of local TMP in hollow fiber obtained experimentally; and secondly, find the proper opportunities for membrane cleaning.

The most widely used monitoring technologies are based on: (1) direct observation technique [15]; (2) direct visualization above the membrane [16]; (3) laser triangulometer [17]; (4) optical laser sensor [18]; (5) ultrasonic time-domain reflectometry [19-21]; (6) NMR imaging [22]; (7) EI spectroscopy [23]; (8) ZP [24]. The EI and ZP can be applied to non-destructive and non-invasive determining local membrane fouling and monitoring fouling behaviors [25].

Previous studies on monitoring the membrane fouling via EI measurements mainly dedicated to the application of dense flat sheet membranes such as reverse osmosis, forward osmosis and ion exchange membranes, but rarely for hollow fiber membranes characterization using EI [23]. EI technique was employed to measure and simulate the fouling of reverse osmosis membrane by Kavanagh et al in 2009 [26]. This work determined electrical properties of the reverse osmosis membrane system. The decrease in the electrical conductivity of the membrane skin layer, which would accompany fouling, is significant at frequencies below $100 \mathrm{~Hz}$. Jia Shin Ho et al. used the EI to monitor biofouling and the electrical properties of fouling process on reverse osmosis membrane in 2016 [27,28]. This study suggests that EIS could be applied "online" using a side-stream, 'canary' cell to continuously monitor a reverse osmosis system to ensure its operations remain below the threshold flux. However, this process has only been reported in recent years, such as Bannwarth et al. have studied the characterization of hollow fiber membrane and cake layer structure using EI in 2015 and 2016 respectively [23,29]. Different from prior study, this article focuses on the investigation of local membrane fouling behaviors and non-uniform fouling process by EI and ZP integration. In the electrical double layer theory of the colloidal system, the potential at the interface between the fixed layer and the diffusion layer is called the ZP [30]. The membrane's charge performance can be determined by measuring the streaming potential of the membrane surface, and the nature and quantity of the adsorbed ions on the membrane surface can be obtained by the theoretical analysis and calculation. This make it possible to characterize membrane fouling from the microscopic perspective.

In recent years, the ZP of membrane as a key parameter have employed by many scholars for determining the membrane fouling mechanisms [31]. Soffer et al. developed a method to determine the fouling of polysulfone ultrafiltration membranes, employing the coupling flux with the ZP [24]. In 2011, Lanteri et al. applied streaming potential coupled with permeate flux measurements for in situ and real time electrokinetic characterization of cakes during dead-end filtration [32]. In 2012, Lanteri et al. also proved that the conversion of tangential electrokinetic measurements into zeta-potential of lumen surface is then no more possible in the hollow fiber membrane filtration [33]. In such cases, it is advisable to carry out streaming current measurements (or to combine streaming potential measurements with electric conductance measurements) because the streaming current is not affected by the cell electric conductance and can then be considered a property of membrane surface. However, only a few studies have determined behaviors of local membrane fouling and non-uniform membrane fouling by in situ EI and ZP which are the electrical measurements.

In this work, the behaviors of local membrane fouling and the development of the non-uniform fouling process were studied by integrating the EI with the ZP. The hydraulic model and equivalent circuit model were employed to improve the solidity of the methods. This study aims to capture the feedback signals of membrane fouling behaviors rapidly using EI and ZP which provide the guidance for HFM module optimization and cleaning on the time and axial space.

\section{Theory}

\subsection{Electrical impedance (EI) spectroscopy}

Based on the Ohm's law, EI can be identified by measuring the amplitude of the applied electrical potential $\hat{U}$, the amplitude of the current $\hat{I}$ and the phase difference $\phi$ under known frequency $\mathrm{f}=\omega / 2 \pi$ [29]:

$Z_{(\omega)}=\frac{U_{(\omega)} \hat{e}^{j(\omega t)}}{I_{(\omega)} e^{\hat{j}(\omega t+\phi)}}=\frac{\hat{U}_{(\omega)}}{\hat{I}_{(\omega)}} e^{-j \phi}=\hat{Z}_{(\omega)} \cos (\phi)-j \hat{Z}_{(\omega)} \sin (\phi)$

Wherein $\hat{Z}_{(\omega)}$ is the amplitude of the EI depending largely on the angular frequency $\omega$, and $\mathrm{j}$ is the imaginary unit. The physical and structural properties of membrane can be further obtained by impedance data measured at a range of frequencies using an equivalent circuit model. If an alternating potential is applied in the measurements, equivalent circuits can be used to simulate the charge transfer of an experimental system.

Such a model can be built up by integrating some different equivalent circuit elements in parallel or in series [23]. These models typically consist of two common circuit elements. The first one is the frequency-independent Ohm's resistance R in which EI can be expressed by the following equation as:

$Z_{R}=R$

The second equivalent circuit element is an ideal capacitor. A capacitor's EI is frequency dependent and can be described as:

$Z_{c}=\frac{1}{j \omega C}$

Wherein $\mathrm{C}$ is the capacitance. The EI was simulated to evaluate the data and to discuss the challenges of EI measuring methods of hollow fiber membranes in a 2-electrode mode. The model employed derive from the model represented by Coster et al. but reduced to one layer due to its homogeneous character as often discussed in UF membranes [34]. This model assumes that current is only conducted either by an ohmic conductance or by an ideal capacitance over the membrane. Consequently, it is not considered the diffusion limitation at the membrane-electrolyte interface or in the membrane pores. If the electrode capacitance $C_{e}$, the membrane capacitance $C_{m}$, and the cake layer capacitance $C_{c-l}$ are placed in parallel, the total capacitance $C_{a c c}$ is simply the sum of all the capacitances.

$\frac{1}{Z C_{a c c}}=\frac{1}{Z C_{e}}+\frac{1}{Z C_{m}}+\frac{1}{Z C_{c-l}}=j \omega \cdot C_{a c c}=j \omega \cdot\left(C_{e}+C_{m}+C_{c-l}\right)$

Approximately, the total conductance $G_{a c c}$ can be calculated if the solution resistance $R_{s}$, the electrode resistance $R_{e}$, the membrane resistance $R_{m}$ and the cake layer resistance $R_{c-l},\left(=\left(\frac{1}{G_{s}}\right)\left(\frac{1}{G_{e}}\right)\left(\frac{1}{G_{m}}\right)\left(\frac{1}{G_{c-l}}\right)\right.$ ) are in parallel. 
$\frac{}{Z_{R_{a c c}}}=\frac{1}{Z_{R_{s}}}+\frac{1}{Z_{R_{e}}}+\frac{1}{Z_{R_{m}}}+\frac{1}{Z_{R_{c-l}}}=G_{s}+G_{e}+G_{m}+G_{c-l}=\frac{1}{R_{a c c}}$

Based on the description above, the mathematical formula of total impedance $Z_{a c c}$ is expressed as:

$Z_{a c c}=R_{s}+Z_{e}+Z_{m}+Z_{c-l}$

Where $Z_{m}$ and $Z_{c-l}$ are the EI of membrane and cake-layer, and $Z_{e}$ and $R_{S}$ are the EI of electrode and bulk solution resistance.

\subsection{The calculation of the $Z P$}

Due to the thermal motion of particles, the charge (ion) in the solution distributes in the adjacent interface solution according to the potential field distribution law, thus forming an electron double layer on the solid-liquid two-phase interface, which is a kind of electrokinetic phenomena caused by the interaction between membrane and solution. It has been found that there is a sliding surface between the dense layer and the diffusion layer. When the surface of the charged solid phase moves relative to the electrolyte solution, a potential difference between the shear surface and the solution phase is generated, which is called zeta potential. Adding pressure in a solution on one side of the membrane results in a potential difference between the two sides of the membrane, which is the steaming potential. The relation between the $\mathrm{ZP}$ and the streaming potential is legitimately given by the classical Helmholtz - Smoluchowski (H-S) Eq. (7) [24]:

$\frac{\Delta E}{\Delta P}=\frac{\varepsilon \varepsilon_{0} \xi}{\mu \lambda}$

Where $\Delta \mathrm{E}$ is the streaming potential difference, $\mathrm{mV} ; \Delta \mathrm{P}$ is the transmembrane pressure, $\mathrm{Pa}$; $\varepsilon$ is the relative dielectric constant of the electrolyte solution; $\varepsilon_{0}$ is the vacuum dielectric constant, $\mathrm{s} / \mathrm{m} \cdot \Omega$; $\xi$ is the zeta potential, $\mathrm{mV} ; \mu$ is the solution viscosity, Pa.s; $\lambda$ is the solution conductivity, $1 / \mathrm{m} \cdot \Omega$. Fig. 1 shows the forming mechanism of ZP.

\subsection{Modification of Hermia's model for constant flux filtration}

The pore blocking laws model having the ability to differentiate the dominant fouling mechanisms by fitting filtration data with the model is often used to analyze membrane fouling. Eq. (8) represents the generalized modality of the blocking laws with model parameters $\mathrm{n}$ and $\mathrm{k}$ representing blocking index and blocking constant, respectively, as studied in Huang et al. [35].

$\frac{d p^{\prime}}{d V_{s}}=k_{v} p^{n}$

Where $P^{\prime}$ is the TMP (P) (Pa) normalized to its initial value $\left(\mathrm{P}_{0}\right)[\mathrm{Pa}]$ and $V_{s}\left[\frac{m^{3}}{m^{2}} \circ r \frac{L}{m^{2}}\right]$ is the unit permeate throughput. This is defined as the cumulative volume of permeate per unit membrane surface area. Table .1 summarizes the values of model parameters $\mathrm{n}$ and $\mathrm{k}$ for the
Table 1

Linear expression of for constant flux filtration based on Hermia's model [35].

\begin{tabular}{lll}
\hline Fouling mechanism & $\mathrm{n}$ & Liner expression \\
\hline Cake layer formation & 0 & $1 / \mathrm{j}_{\mathrm{s}}^{\prime}=1+\mathrm{K}_{\mathrm{v}} \mathrm{V}_{\mathrm{s}}(1)$ \\
Standard blocking & $3 / 2$ & $\mathrm{j}_{\mathrm{s}}{ }^{1 / 2}=1+\mathrm{K}_{\mathrm{v}} / 2 \mathrm{~V}_{\mathrm{s}}(2)$ \\
Intermediate blocking & 1 & $\left.\mathrm{In}_{\mathrm{j}} \mathrm{j}_{\mathrm{s}}^{\prime}\right)=-\mathrm{K}_{\mathrm{v}} \mathrm{V}_{\mathrm{s}}(3)$ \\
Complete blocking & 2 & $\mathrm{j}_{\mathrm{s}}^{\prime}=1-\mathrm{K}_{\mathrm{v}} \mathrm{V}_{\mathrm{s}}(4)$ \\
\hline
\end{tabular}

hypothetical fouling mechanisms as suggested by the blocking laws for dead end constant flux membrane filtration.

The diagrams of the four membrane fouling mechanisms are presented in Fig. 2.

Eq. (8) can be further converted into a function of normalized specific flux, $\mathrm{J}_{\mathrm{s}}^{\prime}$ [dimensionless], by introducing the definition of specific flux: $J_{\mathrm{s}}=\mathrm{J} / \mathrm{P}$ where $\mathrm{J}[\mathrm{m} / \mathrm{s}]$ is the permeate flux and equals permeate flow rate per unit membrane surface area. Thus, the normalized specific flux can be expressed as:

$J_{S^{\prime}}=\frac{J_{s}}{J_{S_{0}}}=\frac{1}{P^{\prime}}$

where $J_{S_{0}}$ is the $J_{S}$ at time zero.

Unlike pore blocking, any changes for the membranes' pore structure did not occur in cake layer filtration stage. On the contrary, the significant increase in membrane hydraulic resistance during HFM filtration was mainly attributed to the formation of the cake layer on the external membrane surface [35]. The application of Darcy's law to cake layer filtration yields:

$\mathrm{P}=\frac{Q \mu\left(R_{m}+R_{c}\right)}{A}$ and $P_{0}=\frac{Q \mu R_{m}}{A}$

Where $\mathrm{Q}$ is permeate flowrate $\left[\mathrm{m}^{3} / \mathrm{s}\right], \mu \mu$ is viscosity [Pa.S $], R_{m}\left[\mathrm{~m}^{-1}\right]$ is the hydraulic resistance of the membrane; and $R_{c}[\mathrm{~m} / \mathrm{kg}]$ is the specific resistance of cake layer. In constant flow filtration mode, $Q$ is constant during filtration and $\mathrm{P}$ increases as a result of membrane fouling.

\section{Materials and methods}

\subsection{Membrane and foulants}

Polyvinylidene fluoride (PVDF) hollow fiber membranes supplied by Tianjin Motimo Membrane Technology Co. Ltd, China, were used in this study. The nominal pore diameter of hollow fiber membranes (PVDF) is $0.2 \mu \mathrm{m}$, inside and outside diameter of $0.6 \mathrm{~mm}$ and $1.1 \mathrm{~mm}$ respectively. Fig. S1 shows the zeta potential of hollow fiber membrane at different $\mathrm{pH}$ measured by a Zeta potential measurement of a solid surface (SurPASS, Anton Paar, Austria). The zeta potential of the hollow fiber membrane was $-12 \pm 0.1 \mathrm{mV}$ at a pH about $7 \pm 0.8$. In order to remove the wetting agent, membranes were immersed in deionized water for at least $24 \mathrm{~h}$.

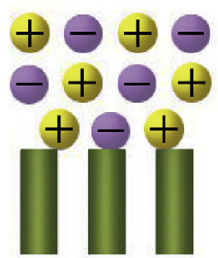

Membrane

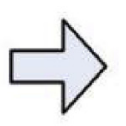

Fig. 1. The schematic diagram of forming mechanism of ZP.

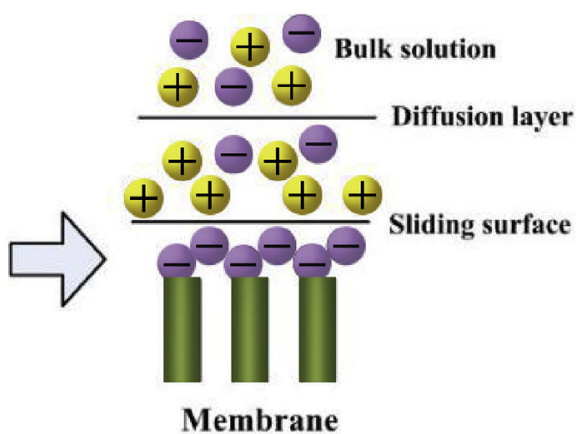



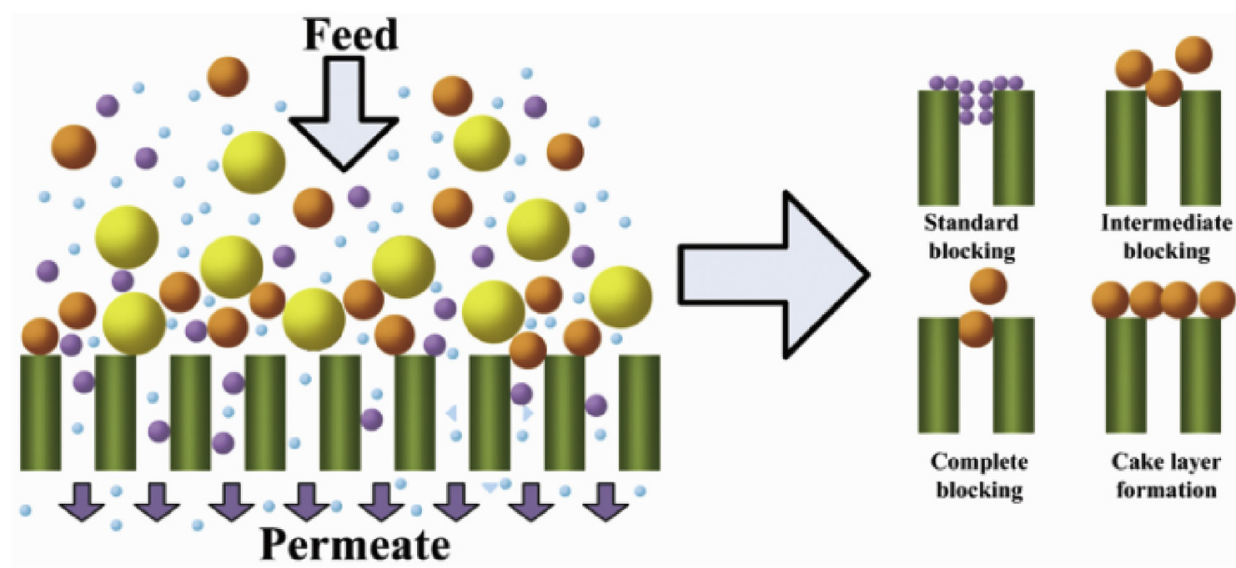

Fig. 2. The schematic diagram of membrane fouling.

Foulant particles were a mixture of yeast (Angel Yeast Co., Ltd., China) cells and kaolin (Superfine, $5 \mu \mathrm{m}$, Aladdin Industrial Co., Ltd., China). Active dry yeast cells and kaolin were dissolved in a $1 \mathrm{mM}$ solution of potassium chloride $(\mathrm{KCl})$ to prepare $0.5 \mathrm{~g} / \mathrm{L}$ feed solution. The mass concentrations of yeast and kaolin were $0.49 \mathrm{~kg} / \mathrm{m}^{3}$ and $0.01 \mathrm{~kg}$ / $\mathrm{m}^{3}$. Fig. S2 shows the zeta potential of feed solution, $0.5 \mathrm{~g} / \mathrm{L}$ kaolin solution and $0.5 \mathrm{~g} / \mathrm{L}$ yeast solution at different $\mathrm{pH}$ measured by ZetaPlus (Zetasizer Nano ZS90, Malvern, UK). The zeta potential of the feed solution was $-0.037 \mathrm{mV}$ at a $\mathrm{pH}$ about $7 \pm 0.8$. This solution had an electrical conductivity of $52.6 \mu \mathrm{S} / \mathrm{cm}$ and a pH about $7 \pm 0.8$ at lab temperatures of $20 \pm 2{ }^{\circ} \mathrm{C}$. Fig. S3 shows the particle size distribution of feed solution, $0.5 \mathrm{~g} / \mathrm{L}$ kaolin solution and $0.5 \mathrm{~g} / \mathrm{L}$ yeast solution measured by a laser scattering particle size distribution instrument (Mastersizer 2000, Malvern, UK). The mean diameter of the feed solution, kaolin solution and yeast solution were $9.538 \mu \mathrm{m}, 3.806 \mu \mathrm{m}$ and $16.493 \mu \mathrm{m}$. Because yeast solution is easier to form aggregates, the particle size is larger when measured separately. Viscosity determined by a viscosimeter (SNB-1, Shanghai Swiss Instrument Co., Ltd., China) was $12 \mathrm{mPa}$.

\subsection{Experiment setup and membrane filtration process}

All filtration experiments were conducted in constant flux dead-end filtration at a flux of $150 \mathrm{~L} /\left(\mathrm{m}^{2} \cdot \mathrm{h}\right)$. All experiments were conducted in outside/in filtration mode. The experimental setup of the filtration experiments illustrated in Fig. 3. Three rectangular water sinks containing the feed solution (the outlet, the middle, the end) were connected in series to simulate an entire hollow fiber membrane. The "outlet" is the fiber initial point which connects the pump. The one far from the constant pressure pump was called the "end". The membrane length and membrane area of each part were $0.3 \mathrm{~m}$ and $2.85 \times 10^{-7} \mathrm{~m}^{2}$ in the filtration experiments, respectively. Membrane permeation was driven by a constant pressure pump (BT100-2J, Longer Pump Co., Ltd., China). The transmembrane pressure (TMP) was monitored by pressure sensors (Danfoss, MBS 3000, Denmark). The values of TMP were automatically recorded by a paperless recorder (MIK210B, MEACON, China) every $5 \mathrm{~min}$. A pair of customized $\mathrm{Ag} / \mathrm{AgCl}$ electrodes were placed on the permeate and retentate sides, respectively. The changes of stream potential between each pair of electrodes were transmitted by a data acquisition system (USB-FS1208, Measurement Computing TM, Hungary), then recorded on a computer using relevant software. The EI were conducted using the an electro-chemical workstation provided by Shanghai Chinstrument Co., Ltd., China, with measurements of hollow fiber membranes conducted in 2-terminal mode. Obviously, the effect of electrode resistance can be eliminated using the 4-terminal mode, which is more conducive to the analysis of experimental results as the reviewer's suggestions, however the 4-terminal mode can not be applied to the analysis of hollow fiber membrane filtration because of very limit space in hollow fiber lumen. Although, the electrode resistance exists in the test results for two-terminal method, no obvious reaction occurs on the electrodes in this experiment, the electrode resistance is constant, which has no influence on the experimental analysis [36]. Thus, twoelectrode system can be used in the experiment.

An electrode pair - a wire electrode and a ring electrode was connected to the electro-chemical workstation in order to measure electrical impedance successfully. The duration of each experiment was $36 \mathrm{~h}$. All experiments were performed in triplicate in the same conditions with the new membranes and solutions to test for repeatability of the experiments. The high resolution electro-chemical workstation served to measure electrical impedance in a range of $0.1-10^{5} \mathrm{~Hz}$. The experimental data were analyzed using "ZsimpWin 3.50 software", which can fit the EI data to the parameters in the equivalent circuit model. In order to ensure the homogeneity of the suspension, the feed solution were stirred periodically and added in to water sinks continuously.

\section{Results and discussion}

\subsection{Local fouling behavior of HFM and the non-uniform fouling process}

As shown in Fig. 4, the TMP, EI and ZP increased rapidly in 0-20 min. During this stage the foulants adsorbed on the membrane surface and blocked the pores, and this increased the filtration resistance, i.e. the TMP increased rapidly. In addition, due to the accumulation and adsorption of foulants on the membrane surface and compaction of electrical double layer, the ZP on the membrane-foulants interface increased rapidly as well. On the other hand, the decreased charge transfer rate resulted in the EI increasing. Thus, the soaring of TMP, EI and ZP in the initial stage could be defined as pore blocking.

The TMP, EI and ZP of three sections increased gradually throughout the duration (20-40 min for the outlet, $20-45 \mathrm{~min}$ for the middle, 20-50 min for the end), shown in Fig. 5. Due to the continuous accumulation of foulants on the membrane surface, cake layer filtration occurred, however, negatively charged foulants and the negatively charged membrane surface existed repulsive effect. This process resulted in the formation of the loose cake layer, and consequently, membrane resistance increased slowly. At this point, the accumulation rate of foulants on the membrane surface was still greater than the outward diffusion rate, which lead to a smooth increase of the ZP. Meanwhile, ions and foulants accumulating on the membrane surface formed the ion-foulant polymers and concentration enhanced the cake layer. Furthermore during this period, the EI gradually increased due to the increase in membrane surface conductance. It can be stated that the period when TMP, EI and ZP gradually increased could be defined as 


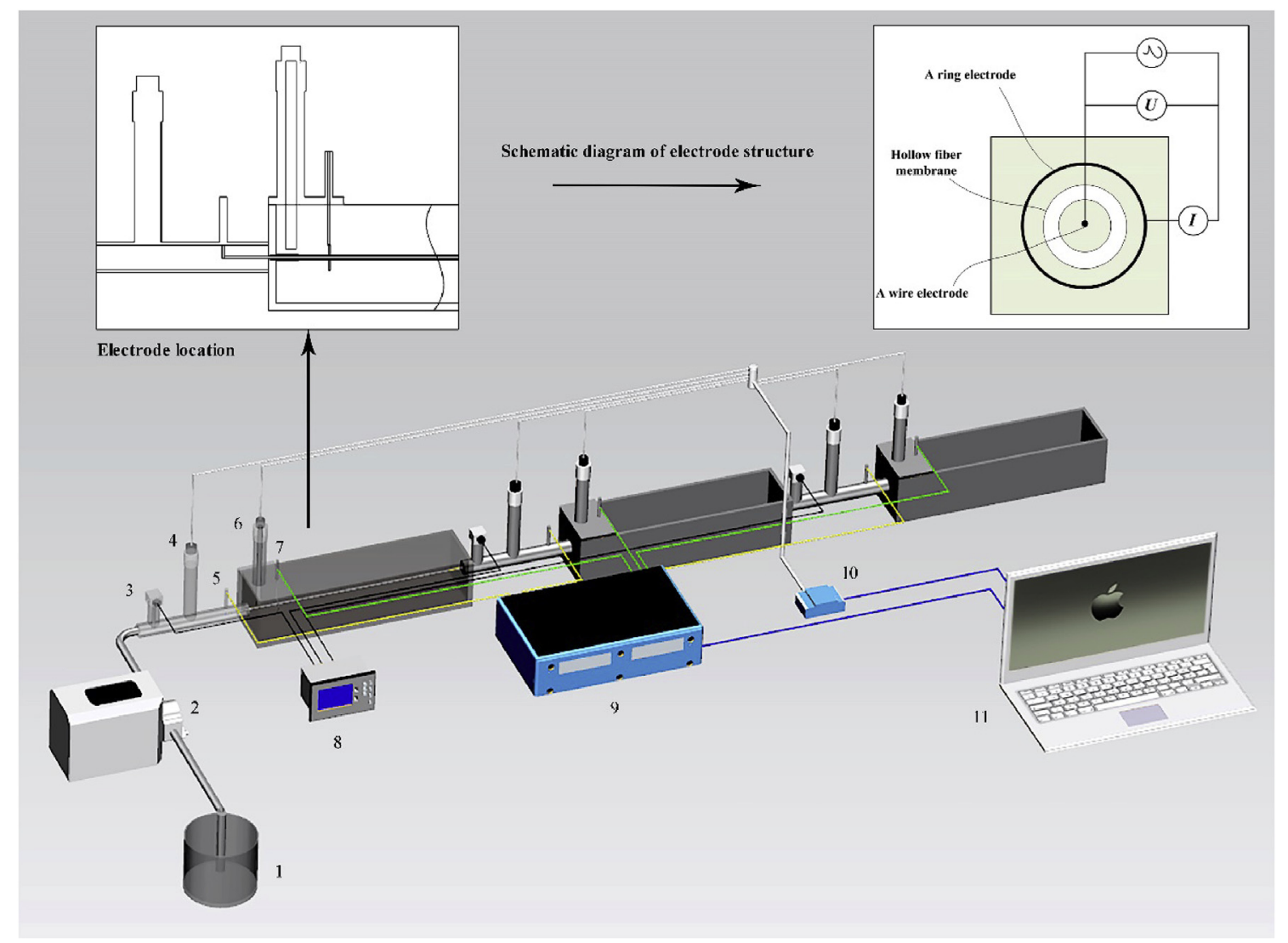

Fig. 3. The diagram of experimental setup in the filtration experiments (1-water sink; 2-pump; 3-pressure sensors; 4,6-Ag/AgCl electrodes; 5-wire electrode; 7-ring electrode; 8-paperless recorder; 9-electro-chemical workstation; 10-data acquisition system; 11- computer).

the process of cake layer formation.

Fig. 6 depicts the changing trends in TMP, EI and ZP as a function of the filtration time $(0-2160 \mathrm{~min})$. At the end of filtration, the filtration resistance increased to a critical state until stability was achieved, and indicated that compaction of cake layer fouling occurred. Simultaneously, the accumulation and diffusion of foulants were balanced and, the amount of ions and foulants on the membrane surface did not increase, which led to the relative stabilization of ZP. In addition, due to cake layer compaction, the bearing capacity of charges between the wire electrode and the ring electrode declined, which resulted in reduced capacitance. The existence of the concentrated polarization was another cause of capacitance reduction. This led to EI decreasing in the cake layer compaction process. Therefore, the decrease in EI but not TMP and ZP could be defined as the cake layer compaction.

Previous studies concluded that local TMP had a strong relationship with local fouling reversibility, namely the bigger the local TMP, then the more serious local membrane fouling became [14]. As shown in Figs. 3-5, the maximum values of TMP, EI and ZP emerged at the outlet of the HFM, indicating that fouling of the outlet were the most serious. The variations of TMP, EI and ZP in the outlet were the first that entered into the stage of cake layer compaction, which was opposite to the end of HFM. This indicated that the non-uniform distribution and difference of fouling proceedings could exist in the HFM process. It could also be deduced that the fibers needed to be cleaned when the outlet entered the cake layer compaction stage. Thus, this work provides the guidance for membrane module optimization and cleaning by analyzing the membrane process in both the time and axial spaces.

In order to verify the previous conclusions in Figs. 3-5, a revision of Hermia's model for constant flux filtration was introduced. The relationship between $P$ and $V_{s}$ was established according to Eq. (8) and Eqs. (1)-(4) in Table .1. The curve of $P$ and $V_{s}$ was fitted linearly at different periods, based on the four objective functions ((1)-(4)) in
Table .1. The measures of fitting $\left(\mathrm{R}^{2} \mathrm{~s}\right)$ and fitted parameters are shown in Figs. S4-S5 of the Supporting Materials. It is worth noting that the value of $R^{2} s$ is adequate for comparing different models under the same operating conditions, but it is inapplicable to compare measures of fitting under different operating conditions [37]. In 0-20 min, the main fouling type of the outlet, the middle and the end $\left(\mathrm{R}^{2}=0.997,0.994,0.994\right.$, respectively) for Eq. (4) was dominated by complete blocking, showing in Fig. S4 of the Supporting Materials. In the different periods (i.e. $20-40 \mathrm{~min}$ for the outlet, $20-45 \mathrm{~min}$ for the middle, 20-50 min for the end), the fitted results were described in Fig. S5 of the Supporting Material. The $\mathrm{R}^{2} \mathrm{~s}(0.999,0.997,0.991)$ of the three sections corresponded to Eq. (1), indicating cake layer formation was the main fouling type. Nonetheless, the model is not suitable for the cake layer compaction. Thus, Darcy's law as depicted in Eq. (10) was introduced to analyze cake layer compaction. As Fig. 7 shows, at the outlet, where the curve slope of the $R_{c}$ increased more rapidly in the filtration time after $40 \mathrm{~min}$ than that of in $20-40 \mathrm{~min}$, indicating cake layer compaction was the main fouling form. The same phenomenon occurred at the middle and the end after $45 \mathrm{~min}$ and $50 \mathrm{~min}$, respectively. These results indicate that the outlet experienced cake layer compaction, followed by the middle and finally the end. When the $R_{c}$ virtually unchanged, this meant that the membrane filtration process was in a balanced state. Therefore, the model confirmed the conclusion reached by analyzing the experimental data of the EI and ZP.

\subsection{The EI data fitting and electrical circuits model}

Fig. 8 shows the bode plot of electrical impedance spectroscopy. Results show that the change in impedance values caused by electrodesolution interface process reflect in the low frequency region (0.1$10^{2} \mathrm{~Hz}$ ). And the membrane-solution interface process and the further development of cake layer lead to the change of impedance in the high 

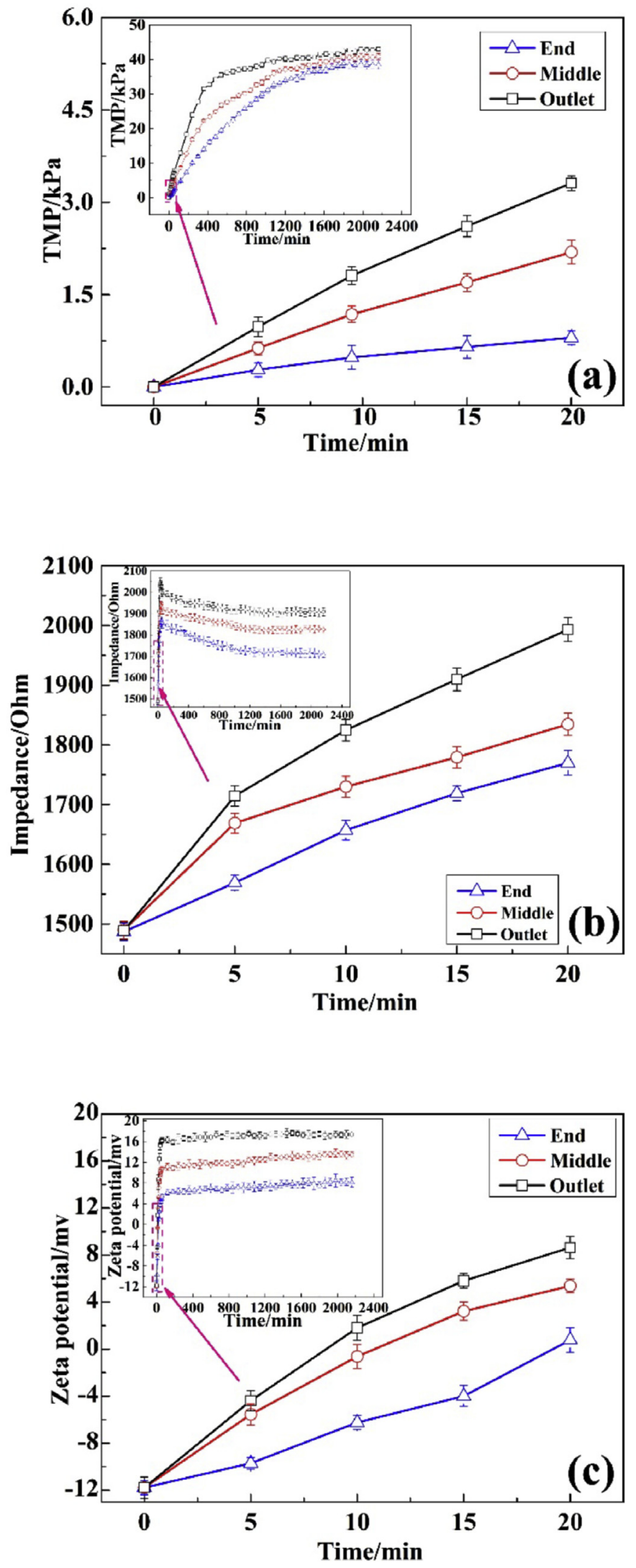

Fig. 4. The variations of measured parameters with the filtration time (0-20 min) during constant flux dead-end filtration of $0.5 \mathrm{~g} / \mathrm{L}$ mixture of yeast and kaolin at $20^{\circ} \mathrm{C}$ : (a) TMP; (b) EI; (c) Zeta potential.
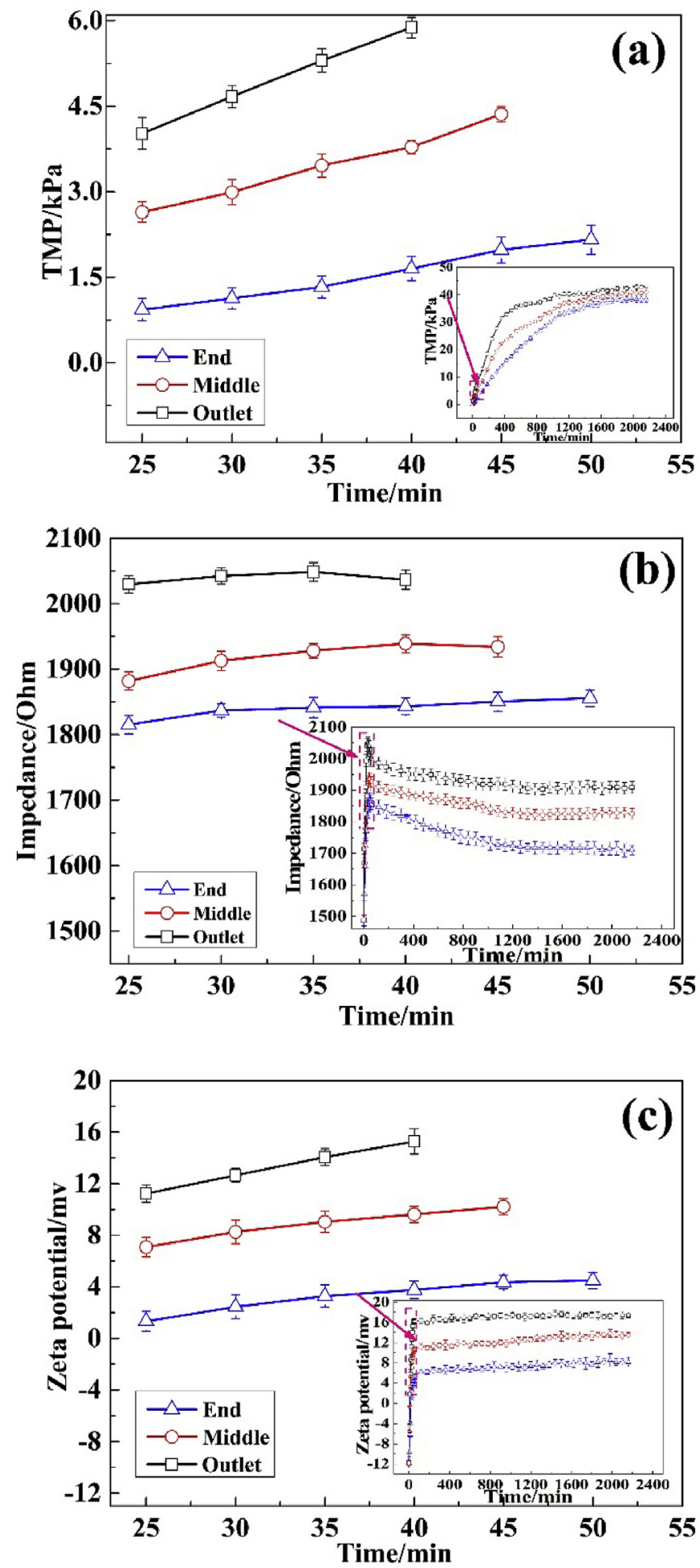

Fig. 5. The variations of measured parameters with the filtration time (20-40 min for the outlet, $20-45 \mathrm{~min}$ for the middle, $20-50 \mathrm{~min}$ for the end) during constant flux dead-end filtration of $0.5 \mathrm{~g} / \mathrm{L}$ mixture of yeast and kaolin at $20^{\circ} \mathrm{C}$ : (a) TMP; (b) EI; (c) Zeta potential.

frequency region (about $10^{2}-10^{5} \mathrm{~Hz}$ ). In the lower frequencies range, the EI increased constantly with a decrease in frequencies. This might attributed to the adsorption of ions in electrolyte on electrode. However, the EI decreased below the plateau area at higher frequencies. This might be resulted from the accumulation of foulant particles which lead to the increase of ion intensity on the membrane surface.

The change in EI calculated by an equivalent circuit model during 

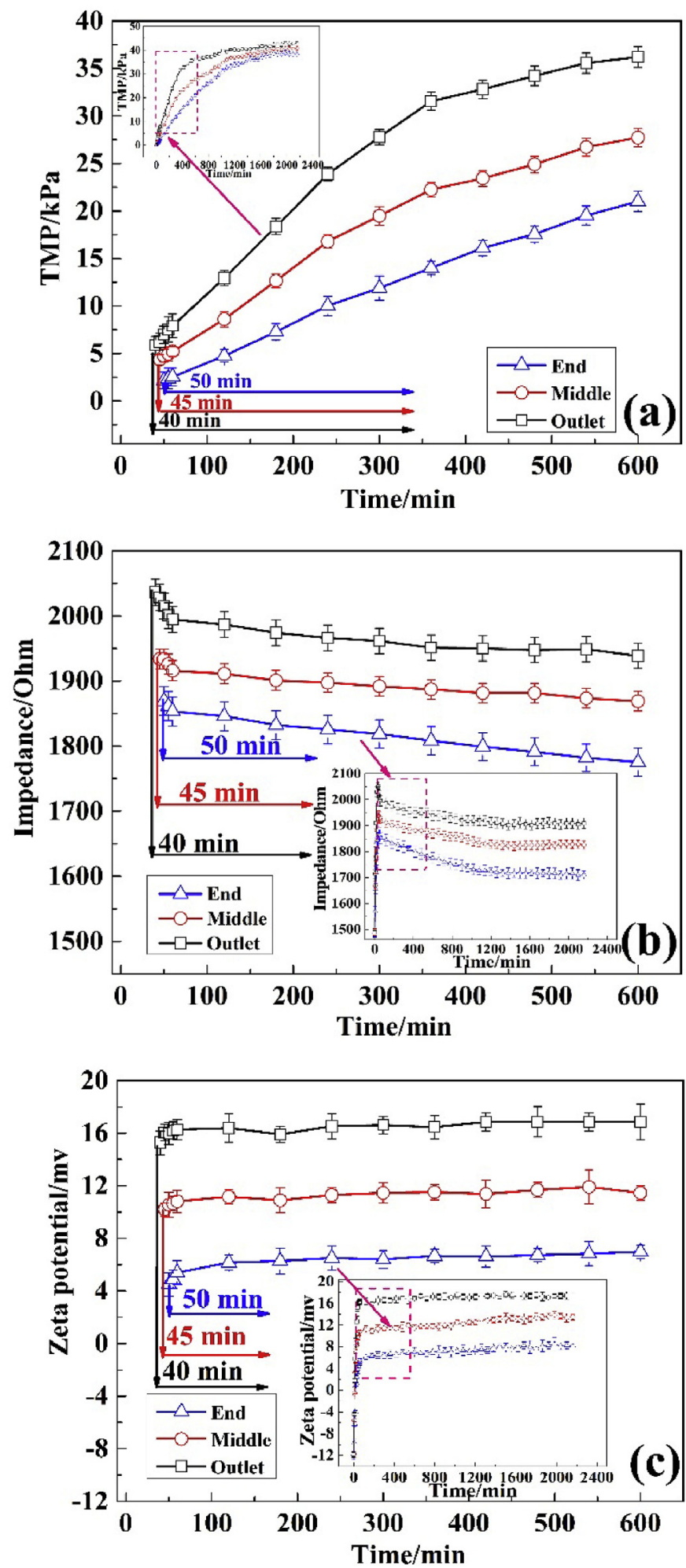

Fig. 6. The variations of measured parameters with the filtration time (40-600 min for the outlet, 45-600 min for the middle, 50-600 min for the end) during constant flux dead-end filtration of $0.5 \mathrm{~g} / \mathrm{L}$ mixture of yeast and kaolin at $20^{\circ} \mathrm{C}$ : (a) TMP; (b) EI; (c) Zeta potential.

membrane filtration was depicted in Fig. 9. Actually, the equivalent circuit model could describe the major electro-chemical contributions in all the compartments. The (a) of Fig. 9 shows an equivalent circuit model before the formation of the cake layer while the (b) depicted the equivalent circuit model after the formation of cake layer. Solution

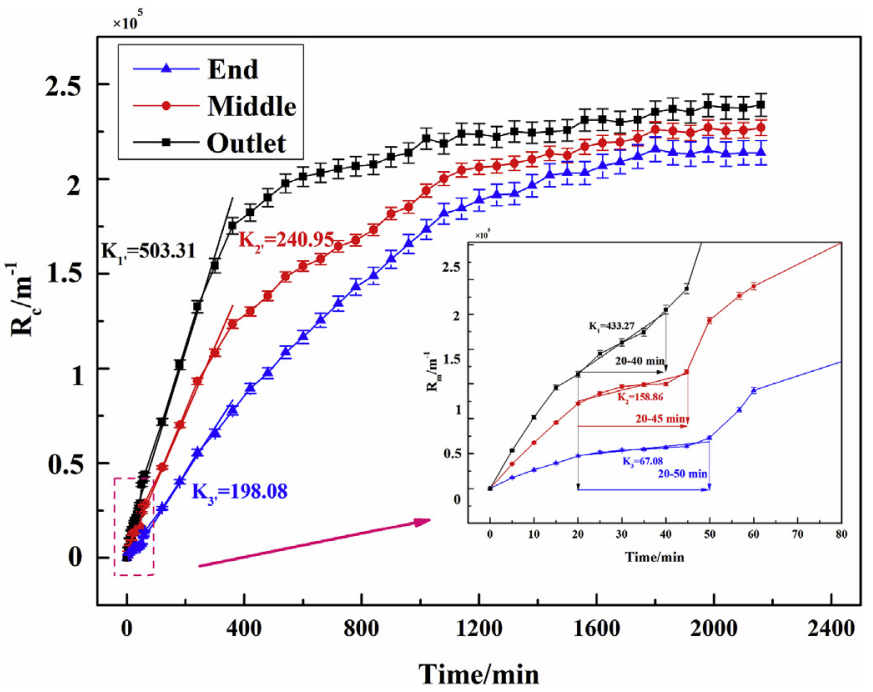

Fig. 7. The variations of Rc with the filtration time $(0-2160 \mathrm{~min})$ during constant flux dead-end filtration of $0.5 \mathrm{~g} / \mathrm{L}$ mixture of yeast and kaolin at $20^{\circ} \mathrm{C}$.

resistance, membrane resistance, membrane capacitance, electrode resistance, and electrode capacitance existed in the filter system at the beginning of filtration. However, compared with the initial stage, the equivalent circuit was more than two circuit elements i.e. cake layer resistance and cake layer capacitance. And the simulation results of three sections were consistent. The first resistance was the one of the feed solution and the cables. Their contribution to the total resistance could be represented by an ohmic resistance and a capacitor in parallel.

Fig. 10 illustrates some fitted values in the three sections. According to the simulation data, it is worth mentioning that the cake layer capacitances during cake layer compaction were significantly smaller than the cake layer capacitances in the cake layer formation period. The results meant that the bearing capacity of charges between the wire electrode and the ring electrode decreased. Thus the correctness of the previous analysis shown in Fig. 9 is confirmed. Notably, $R_{m}$ of the outlet in the cake layer formation significantly increased compared with the value in the cake layer compaction, indicating that irreversible fouling could be most serious during cake layer compaction. The middle and the end experienced the same phenomenon. Consequently, this was the right time to clean the membrane and minimize the occurrence of irreversible fouling. The $\mathrm{R}_{\mathrm{c}-1}$ and $\mathrm{C}_{\mathrm{c}-1}$ of the outlet was maximum, indicating the outlet's membrane fouling of the outlet was the most serious. However, this result was in contrast to the end of HFM. This evidence proved that the non-uniform distribution and difference of fouling proceedings could exist in the HFM process.

\section{Conclusion}

This paper proposed a theoretical research method for cake layer compaction and put forward a membrane fouling control method based on the spatio-temporal variation of hollow fiber membrane fouling. The specific findings are as follows:

- The behavior of local membrane fouling could successfully be monitored in-situ by the EI and the ZP. Integrating the results with the hydraulic model and equivalent circuit model confirmed the accuracy of the results.

- During the initial filtration, the TMP, EI and ZP increased rapidly, which corresponded to the pore blocking evidence. Referring to the medium of filtration, the TMP, EI and ZP gradually increased which suggested that cake layer formation dominated the fouling process. The characteristics of non-uniform fouling of the HFM are reflected in the spatio-temporal difference during the fouling process. In other 


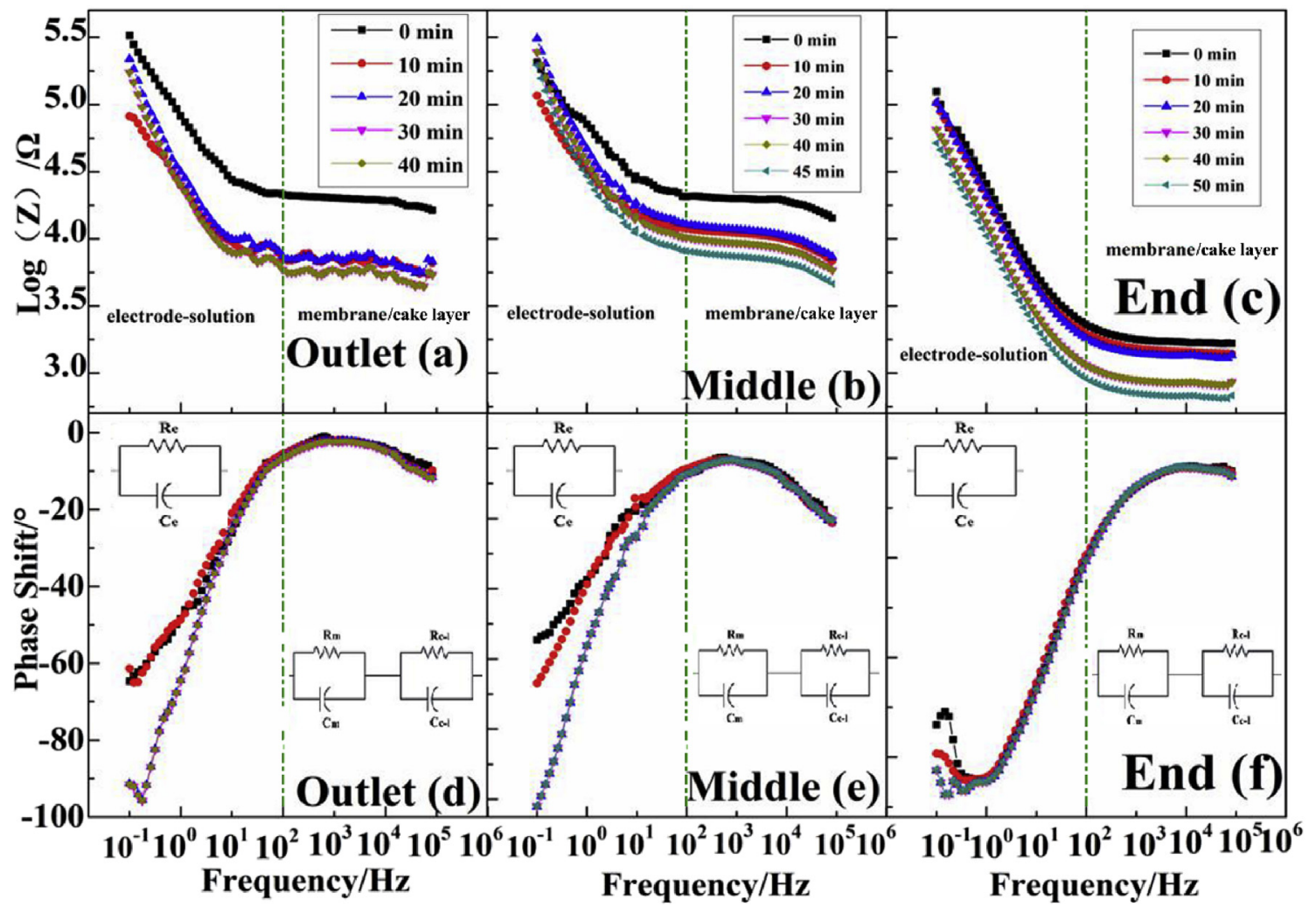

Fig. 8. The bode plot of three sections at different moment during constant flux dead-end filtration of $0.5 \mathrm{~g} / \mathrm{L}$ mixture of yeast and kaolin at $20^{\circ} \mathrm{C}$. (a) and (d): the Log (Z) and phase shift with the frequency $\left(10^{-1}-10^{5}\right)$ of the outlet; (b) and (e): the $\log (\mathrm{Z})$ and phase shift with the frequency $\left(10^{-1}-10^{5}\right)$ of the middle; (c) and (f): the $\log (\mathrm{Z})$ and phase shift with the frequency $\left(10^{-1}-10^{5}\right)$ of the end.

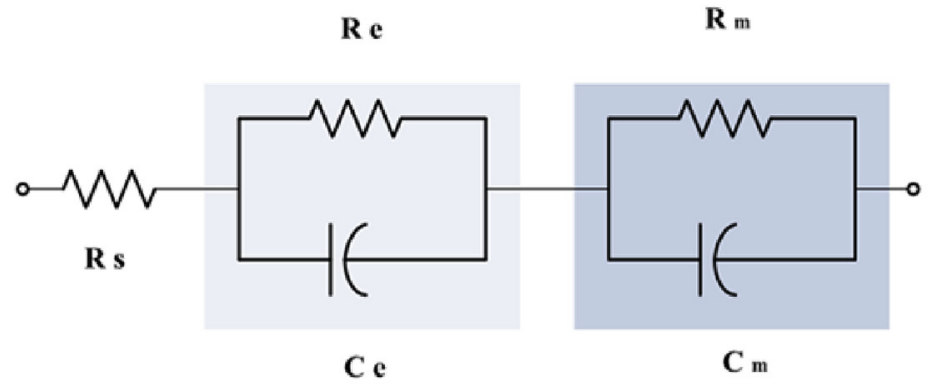

(a)

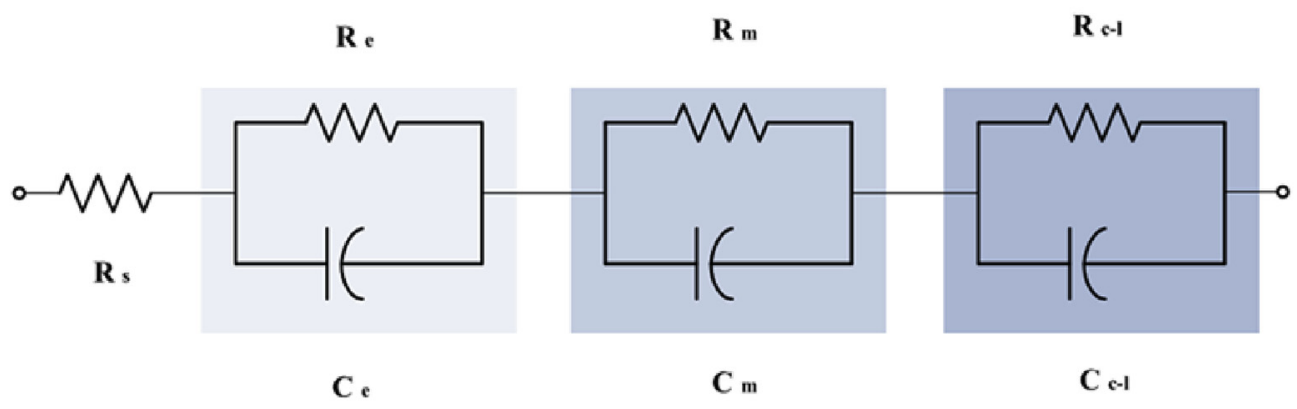

(b)

Fig. 9. Schematic diagram of fitting electrical circuits at different moment according to experimental data using "ZsimpWin 3.50 software": (a) 0 min (b) 20 min. 


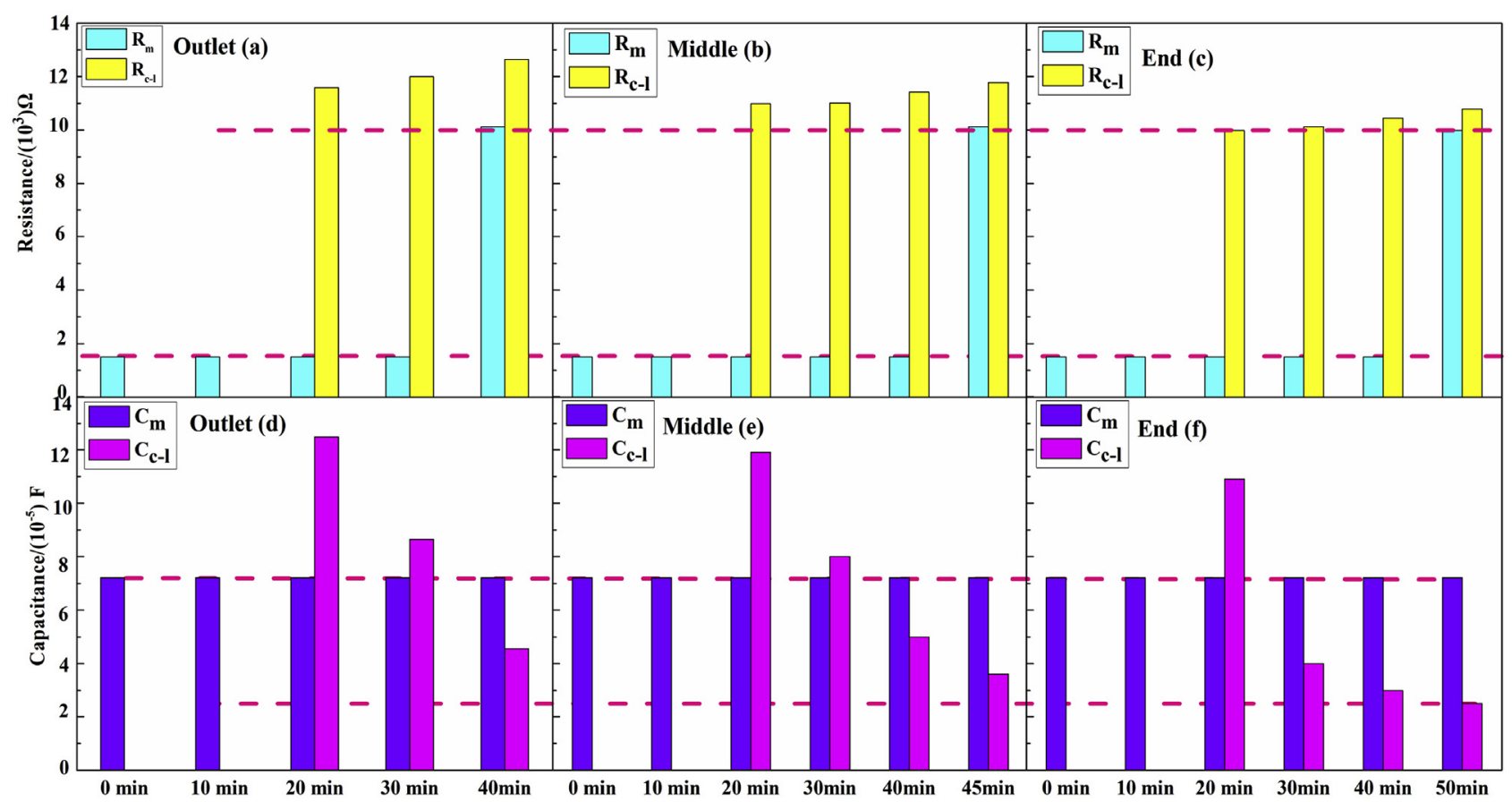

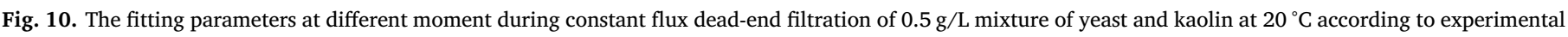

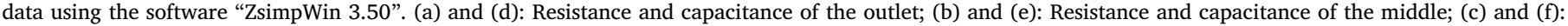
Resistance and capacitance of the end.

words, the location with the greater pressure is where the more serious membrane fouling occurred, and this is when it entered the cake layer compaction more quickly.

- The revision of Hermia's model for constant flux filtration and Darcy's Law was employed to verify the filtration process. A comprehensive equivalent circuit model was developed to analyze the changes in EI during filtration process. These methods can be applied to in-depth investigation of different types of membrane fouling and non-uniform distribution of local membrane fouling.

\section{Acknowledgements}

This study is financially supported by the National Natural Science Foundation of China (No.51638011,51578375), Science and Technology Planning Project of Tianjin, China (14ZCDGSF00128,16PTGCCX00070), and Program for Innovative Research Team in University of the Ministry of Education of China (Grant no. IRT-17R80). The authors would thank for the supports of China Scholarship Council (No. 201709345009, No.201606250111).

\section{Appendix A. Supplementary data}

Supplementary data to this article can be found online at https:// doi.org/10.1016/j.memsci.2019.117245.

\section{References}

[1] Y. Zhang, J. Wang, F. Gao, Y.S. Chen, H.W. Zhang, A comparison study: the different impacts of sodium hypochlorite on PVDF and PSF ultrafiltration (UF) membranes, Water Res. 109 (2017) 238-247.

[2] F. Gao, J. Wang, H.W. Zhang, H. Jia, Zh Cui, G. Yang, Aged PVDF and PSF ultrafiltration membranes restored by functional polydopamine for adjustable pore sizes and fouling control, J. Membr. Sci. (2019) 156-167 570-571.

[3] Zh Cui, J. Wang, H.W. Zhang, L.F. Song, H. Jia, G. Yang, F. Gao, Influence of selective permeation of backwashing solution on the cleaning effectiveness in hollow fiber system, J. Membr. Sci. 546 (2018) 139-150.

[4] Y.F. Wang, H. Jia, J. Wang, B.A. ChengG, Yang F. Gao, Impacts of energy distribution and electric field on membrane fouling control in microbial fuel cellmembrane bioreactor (MFC-MBR) coupling system, Bioresour. Technol. 269 (2018)
339-345

[5] S. Chang, A.G. Fane, The effect of fibre diameter on filtration and flux distribution relevance to submerged hollow fibre modules, J. Membr. Sci. 184 (2001) 221-231.

[6] S. Liang, Y.B. Zhao, J. Zhang, L.F. Song, Bisection method for accurate modeling and simulation of fouling in hollow fiber membrane system, Environ. Sci. Pollut. Res. 24 (2017) 14346-14354.

[7] X.H. Li, J.X. Li, J. Wang, H. Wang, B.Q. He, H.W. Zhang, W.S. Guo, H.H. Ngo, Experimental investigation of local flux distribution and fouling behavior in doubleend and dead-end submerged hollow fiber membrane modules, J. Membr. Sci. 453 (2014) 18-26.

[8] S. Chang, A.G. Fane, S. Vigneswaran, Modeling and optimizing submerged hollow fiber membrane modules, AIChE J. 48 (2002) 2203-2212.

[9] C. Tien, R. Bai, An assessment of the conventional cake filtration theory, Chem. Eng. Sci. 58 (2003) 1323-1336.

[10] C. Tien, B.V. Ramarao, Revisiting the laws of filtration: an assessment of their use in identifying particle retention mechanisms in filtration, J. Membr. Sci. 383 (2011) $17-25$.

[11] P. Le-Clech, V. Chen, T.A. Fane, Fouling in membrane bioreactors used in wastewater treatment, J. Membr. Sci. 284 (2006) 17-53.

[12] A. Grenier, M. Meireles, P. Aimar, P. Carvin, Analysing flux decline in dead-end filtration, Chem. Eng. Res. Des. 86 (2008) 1281-1293.

[13] S.H. Yoon, S.H. Lee, I.T. Yeom, Experimental verification of pressure drop models in hollow fiber membrane, J. Membr. Sci. 310 (2008) 7-12.

[14] W.N. Lee, W.S. Cheong, K.M. Yeon, B.K. Hwang, C.H. Lee, Correlation between local TMP distribution and bio-cake porosity on the membrane in a submerged MBR, J. Membr. Sci. 332 (2009) 50-55.

[15] H. Li, A. Fane, H. Coster, S. Vigneswaran, Direct observation of particle deposition on the membrane surface during crossflow microfiltration, J. Membr. Sci. 149 (1998) 83-97.

[16] A. Subramani, E.M. Hoek, Direct observation of initial microbial deposition onto reverse osmosis and nanofiltration membranes, J. Membr. Sci. 319 (2008) 111-125.

[17] J. Altmann, S. Ripperger, Particle deposition and layer formation at the crossflow microfiltration, J. Membr. Sci. 124 (1997) 119-128.

[18] M. Hamachi, M. Mietton-Peuchot, Cake thickness measurement with an optical laser sensor, Chem. Eng. Res. Des. 79 (2001) 151-155.

[19] X. Xu, J. Li, H. Li, Y. Cai, Y. Cao, B. He, Y. Zhang, Non-invasive monitoring of fouling in hollow fiber membrane via \{UTDR\}, J. Membr. Sci. 326 (2009) 103-110.

[20] J. Li, R. Sanderson, In situ measurement of particle deposition and its removal in microfiltration by ultrasonic time-domain reflectometry, Desalination 146 (2002) 169-175.

[21] S. Sim, T. Chong, W. Krantz, A. Fane, Monitoring of colloidal fouling and its associated metastability using ultrasonic time domain reflectometry, J. Membr. Sci. 401 (2012) 241-253.

[22] S. Bütehorn, L. Utiu, M. Küppers, B. Küppers, T. Wintgens, M. Wessling, T. Melin, NMR imaging of local cumulative permeate flux and local cake growth in submerged microfiltration processes, J. Membr. Sci. 371 (2011) 52-64.

[23] S. Bannwarth, T. Trieu, C. Oberschelp, M. Wessling, On-line monitoring of cake layer structure during fouling on porous membranes by in situ electrical impedance 
analysis, J. Membr. Sci. 503 (2016) 188-198.

[24] Y. Soffer, J. Gilron, A. Adin, Streaming potential and SEM-EDX study of UF membranes fouled by colloidal iron, Desalination 146 (2002) 115-121.

[25] M. Sluyters-Rehbach, Impedances of electrochemical systems - terminology, nomenclature and representation.1.Cells with metal-electrodes and liquid solutions, Pure Appl. Chem. 66 (1994) 1831-1891.

[26] J.M. Kavanagh, S. Hussain, T.C. Chilcott, H.G.L. Coster, Fouling of reverse osmosis membranes using electrical impedance spectroscopy: measurements and simulations, Desalination 236 (2009) 187-193.

[27] J.S. Ho, J.H. Low, L.N. Sim, R.D. Webster, S.A. Rice, A.G. Fane, H.G.L. Coster, Insitu monitoring of biofouling on reverse osmosis membranes: detection and mechanistic study using electrical impedance spectroscopy, J. Membr. Sci. 518 (2016) 229-242.

[28] J.S. Ho, L.N. Sim, J. u, R.D. Webster, A.G. Fane, H.G.L. Coster, A threshold flux phenomenon for colloidal fouling in reverse osmosis characterized by transmembrane pressure and electrical impedance spectroscopy, J. Membr. Sci. 500 (2016) $55-65$.

[29] S. Bannwarth, M. Darestani, H. Coster, M. Wessling, Characterization of hollow fiber membranes by impedance spectroscopy, J. Membr. Sci. 473 (2015) 318-326.

[30] B. Teychene, P. Loulergue, C. Guigui, et al., Development and use of a novel method for in line characterisation of fouling layers electrokinetic properties and for fouling monitoring, J. Membr. Sci. 370 (2011) 45-57.
[31] S. Schiewer, B. Volesky, Modeling of the protonmetal ion exchange in biosorption, Environ. Sci. Technol. 29 (1995) 3049-3058.

[32] Y. Lanteri, P. Fievet, C. Magnenet, S. Déon, A. Szymczyk, Electrokinetic characterisation of particle deposits from streaming potential coupled with permeate flux measurements during dead-end filtration, J. Membr. Sci. 378 (2011) 224-232

[33] Y. Lanteri, P. Fievet, S. DéoN, P. Sauvade, W. Ballout, A. Szymczyk, Electrokinetic characterization of hollow fibers by streaming current, streaming potential and electric conductance, J. Membr. Sci. 411-412 (2012) 193-200.

[34] H.G.L. Coster, K.J. Kim, K. Dahlan, J.R. Smith, C.J.D. Fell, Characterization of ultrafiltration membranes by impedance spectroscopy.1. Determination of the separate electrical parameters and porosity of the skin and sublayers, J. Membr. Sci. 66 (1992) 19-26.

[35] H. Huang, T.A. Young, J.G. Jacangelo, Unified membrane fouling index for low pressure membrane filtration of natural waters: principles and methodology, Environ. Sci. Technol. 42 (2008) 714-720.

[36] F. Gao, J. Wang, H.W. Zhang, H. Jia, Zh Cui, G. Yang, Role of ionic strength on protein fouling during ultrafiltration by synchronized UV-vis spectroscopy and electrochemical impedance spectroscopy, J. Membr. Sci. 563 (2018) 592-601.

[37] Y. Zhang, J. Wang, F. Gao, H. Tao, Y.S. Chen, H.W. Zhang, Impact of sodium hypochlorite (NaClO) on polysulfone (PSF) ultrafiltration membranes: the evolution of membrane performance and fouling behavior, Separ. Sci. Technol. 175 (2017) 238247. 